\title{
The Influence of Hormonal Disorders on Quality of Life of Women with Acne
}

\author{
Bogomolets National Medical University, Kyiv, Ukraine \\ Liudmyla Naumova MD
}

\section{Introduction}

About $80 \%$ of people had acne of some form. This dermatosis manifests at age $12-14$, when there is a physiological increase in the level of hormones, and in most cases it regresses at 18-22. However today, the number of women with acne at age 25 and older has significantly increased.

\section{Materials and methods}

Conducted was prospective observational study involving 126 women aged 19 to 37 years with inflammatory form of acne of different severity. All of the women were studied for blood hormonal level in order to detect the syndrome of hyperandrogenism. Quality of Life Cardiff Acne Disability Index (CADI) was used in order to assess the quality of life of patients.

\section{Results}

Among the examined 126 women, condition of acne was mild in $42 \%$ of cases, moderate in $51 \%$, and severe in $7 \%$. According to the level of hormone concentrations in blood, syndrome of hyperandrogenism was determined in 109 of studied females. Adrenal genesis of hyperandrogenism was established in $44.9 \%$ of women, mixed genesis - in 34.9\%, and ovarian genesis - in 20.2\%. Among the women of this research, the mean score on the CADI responses was 7.8 , and $84.1 \%$ of them had the CADI score more than 6 , indicating a marked impact on quality of life. The mean score of CADI in women with adrenal type of hyperandrogenism was 6.2 , with ovarian type -7.3 , and with mixed type - 9.8 .

\section{Conclusions}

It was found out that $86.5 \%$ of adult women with acne had different hormonal disorders that lead to development of acne. It was also found out that the quality of life of women with acne is influenced not only by the severity of the clinical manifestation and localization of skin rashes, but an important role is played by the genesis of hyperandrogenism. 